\title{
Fées, nourrices et superstitions : les soins aux nourrissons au prisme de la fiction médiévale
}

Fairies, wet nurses and superstitions: looking after toddlers through the prism of medieval fiction

\section{Yasmina Foehr-Janssens}

\section{CpenEdition}

\section{Journals}

Édition électronique

URL : http://journals.openedition.org/abpo/3698

DOI : $10.4000 /$ abpo.3698

ISBN : 978-2-7535-6539-5

ISSN : 2108-6443

Éditeur

Presses universitaires de Rennes

Édition imprimée

Date de publication : 16 novembre 2017

Pagination : 109-133

ISBN : 978-2-7535-5674-4

ISSN : 0399-0826

Référence électronique

Yasmina Foehr-Janssens, «Fées, nourrices et superstitions : les soins aux nourrissons au prisme de la fiction médiévale ", Annales de Bretagne et des Pays de l'Ouest [En ligne], 124-3 | 2017, mis en ligne le 16 novembre 2019, consulté le 03 janvier 2020. URL : http://journals.openedition.org/abpo/3698 DOI : 10.4000/abpo.3698 


\title{
Fées, nourrices et superstitions : les soins aux nourrissons au prisme de la fiction médiévale
}

\author{
Yasmina FOEHR-JANSSENS \\ Professeure de littérature française médiévale, université de Genève, \\ groupe de recherche "Lactation in history "
}

Sous ce titre, je présenterai un travail en cours dans le cadre d'un programme de recherche financé par le fonds national de la recherche scientifique suisse (FNRS) et consacré aux représentations et aux pratiques de l'allaitement dans la longue durée. Le projet "Lactation in history " se propose d'étudier l'allaitement comme une réalité historique et culturelle complexe. Il s'agit de mettre en évidence comment les discours, les représentations et les pratiques de la lactation et de l'alimentation des nourrissons se développent et évoluent de l'Antiquité jusqu'à aujourd'hui. Loin de se limiter à un soi-disant "monde des nourrices ", clos sur lui-même et sans rapport avec la sphère publique, ceux-ci prennent des significations religieuses et politiques qui varient en fonction des différents contextes culturels étudiés. À toute époque, l'allaitement fait l'objet d'une surdétermination symbolique. La lactation sert de support à un imaginaire de la puissance fécondante dont les diverses mises en scène éclairent la construction des rapports sociaux de sexe.

Il s'agira plus précisément de considérer les soins prodigués aux nourrissons sous l'angle de leur représentation fictionnelle dans la littérature française du Moyen Âge. Ce parti pris permet de montrer que, contrairement à un certain nombre d'idées reçues, les grands genres narratifs médiévaux, le roman et la chanson de geste, accordent une attention soutenue aux faits et gestes qui entourent la venue au monde des humains Les mentions des rites et des pratiques liés à la naissance et à la petite enfance ne forment pas un ensemble de notations éparses et sans signification générale, ni liens entre elles. Elles offrent, lorsqu'on y regarde de près, un panorama imaginaire très précis concernant les enjeux de la reproduction humaine, dans sa composante sociale, notam- 
ment ${ }^{1}$. Les traditions romanesque et épique médiévales s'attachent à formuler, en termes esthétiques aussi bien qu'éthiques, les aspirations à la gloire de la classe chevaleresque. Cette entreprise d'autocélébration est toutefois bien souvent passée au crible de la critique cléricale, en particulier dans le cas des romans, ce qui confère une complexité véritable et une grande polyphonie aux œuvres que nous étudions. La fabrique du héros prend, en particulier au cours du XIII ${ }^{\mathrm{e}}$ siècle et au XIV ${ }^{\mathrm{e}}$ siècle, un tour particulier. Tout un appareil de motifs merveilleux, d'origine folklorique aussi bien qu'hagiographique, vient s'attacher à la description des soins et de l'accueil prodigués aux nourrissons, leur conférant une aura féerique qui confine à la mise en place d'une sorte de mythologie aux contours certes indécis, mais dominée par l'action de puissantes figures nourricières dont il convient de déterminer la fonction, qui peut varier d'un texte à l'autre.

\section{Les nourrices en littérature : complicité féminine, prescriptions masculines et vulnérabilité}

\section{La nourrice breneuse : hostilité et ignorance}

À première vue pourtant, une telle valorisation ne va pas tout à fait de soi. Un relevé des occurrences de la figure de la nourrice dans la littérature médiévale fait apparaître de nombreuses mentions peu flatteuses de ce personnage qui occupe une place très secondaire dans les récits. Son apparition dans les œuvres littéraires semble rare et modeste, le plus souvent très anecdotique, comme en témoigne, à titre d'exemple, un passage du Roman de Tristan en prose qui accorde une attention plus que superficielle aux activités liées à la puériculture :

"Il li firent querre norrice por li alaitier et por faire tele norreture come a tel enfant apartient. Mes atant lesse ore li contes a parler de Tristan et dou roi Melyadus, son pere $[\ldots]^{2}$."

["Ils firent chercher une nourrice pour l'allaiter et lui procurer la nourriture qui convient à un enfant de sa qualité. Mais désormais le conte cesse de parler de Tristan et du roi Melyadus, son père. "]

1. Ces constatations convergent avec les travaux des historiens qui ont revalorisé l'importance de l'enfance au Moyen Âge, voir ALEXANDRE-BIDON, Danièle, L'enfant à l'ombre des cathédrales, Lyon, Presses universitaires de Lyon, 1985; Riché, Pierre, AlEXANDREBIDON, Danièle, L'enfance au Moyen Âge, Paris, Seuil, 1994; ALEXANDRE-BIDON, Danièle, LETT, Didier, Les Enfants au Moyen Âge, Paris, Hachette, 1997; LETT, Didier, L'enfant des miracles : enfance et société au Moyen Âge, Paris, Aubier, 1997.

2. Le Roman de Tristan (XIII ${ }^{\mathrm{e}}$ siècle), éd. R. L. CuRTIS, Munich, Max Hueber Verlag, 1963, t. I, p. 129. Nous présentons les données rapidement résumées ici dans un article à paraître dans les actes du colloque de l'INED (Paris, 25-26 mars 2014), Allaitement et pratiques de sevrage : approches pluridisciplinaires et diachroniques : FOEHR-JANSSENS, Yasmina, Roux, Brigitte, VENTURI, Céline, «Représentations de l'allaitement au Moyen Âge : invisibilité ou prolifération matérielle et légendaire ". 
Bien plus, la nourrice se présente sous un jour particulièrement peu recommandable. Elle est, aux yeux des clercs qui évoquent sa présence, la protagoniste par excellence d'un univers féminin caractérisé par l'ignorance, la superstition, la malice et l'hostilité à l'égard des hommes et plus particulièrement des hommes mariés. Ces préjugés sont plus particulièrement relayés par la tradition de textes antiféministes ou misogames. Les occupations de la nourrice font l'objet de descriptions assez précises, bien qu'elles soient prises le plus souvent en mauvaise part. Un texte parodique de la fin du XIV ${ }^{\mathrm{e}}$ siècle ou du début du XV $\mathrm{XV}^{\mathrm{e}}$ siècle, les Quinze Joies de mariage, décrit par le menu les calamités qui s'abattent sur la vie domestique d'un homme sitôt qu'il se prend dans la " nasse " du mariage. La nourrice et les sages-femmes, matrones ou ventrières, appartiennent à une faction malveillante qui entretient et attise l'inimitié de l'épouse envers son époux afin de priver celui-ci de tout repos :

"Puis s'en va coucher, et oyt les enfans crier la nuitée; et la dame et la nourrice les lessent à l'aventure crier tout à escient, par despit du bon-homme. Ainsi passe la nuyt en soussy et tourmens ${ }^{3}$."

[" Ensuite, il va se coucher et entend les enfants crier toute la nuit; parfois la femme et la nourrice les laissent crier exprès pour le faire enrager. C'est ainsi qu'il passe la nuit dans les soucis et les tourments ${ }^{4}$. "]

Les soins à donner aux nourrissons mettent la nourrice en contact avec les langes qu'il faut changer et laver. L'idée de souillure induite par ces tâches peu ragoûtantes s'exprime dans la Farce des chambrières. Les servantes se plaignent d'une " orde vieille nourrice " qu'elles traitent de " breneuse ". La présence d'un enfant dans la maison les oblige à aller à la rivière laver " un tas de drappeaulx pleins de fi fi à gros morceaux " :

\author{
" PREMIERE CHAMBERIERE \\ Je n'ay en ce monde tempeste \\ Qui me desplaise en mon service \\ Que d'une orde vielle nourrice \\ Qui nourit ung petit enfant \\ De l'ostel. \\ LA SECONDE \\ Ha! je les hays tant : \\ Ces nourices tant flateresses \\ Sont tousjours auprès des maistresses, \\ C'est une chose qui me fasche. \\ [...] \\ LA SECONDE \\ Ce n'est que ordure : \\ Il y a ung tas de drappeaux
}

3. Les quinze Joies de mariage, publié par Jean RYCHNER, Genève, Droz, 1963, Quarte Joye, p. 32.

4. Les quinze joies de mariage, traduites et présentées par Monique SANTUCCI, Paris, Stock, coll. « Moyen Âge ", 1986, p. 53. 
Plains de fy fy à gros morceaux,

Cela me fait tant de mal au cueur.

LA PREMIÈRE

Cela me fait plus de douleur

Qu'il fault aller à la rivière

Et en estre la chamberière

De ceste nourrice breneuse

Et si encore est envieuse

Des gens ${ }^{5}$."

" [PREMIËRE SERVANTE :

Aucune calamité en ce monde ne me déplait autant dans mon service qu'une vielle nourrice dégoutante qui nourrit le petit enfant dans cette maison.

LA SECONDE :

Ah! Je les hais tant. Ces nourrices sont des flatteuses qui accaparent les maîtresses. Voilà une chose qui me fâche.

[...]

LA SECONDE :

Ce n'est que saleté. Il y a un tas de langes pleins de gros morceaux de fiente.

Cela me donne la nausée.

LA PREMIÈRE :

Cela m'est d'autant plus pénible qu'il faut aller à la rivière et servir cette nourrice merdeuse qui, en plus, est très envieuse. "]

Dans un registre tout différent, mais qui se révèlera d'un grand intérêt pour notre enquête, la nourrice peut incarner la figure du mauvais littérateur, selon une tradition présente au début du Commentaire au songe de Scipion de Macrobe. Dans son premier chapitre, l'auteur du ve siècle s'intéresse à la valeur de vérité que l'on peut reconnaître à la fiction. Pour ce faire, il met en place une typologie des genres fabuleux. La catégorie la moins recommandable, celle des récits sans aucune valeur, ni morale, ni philosophique, n'a d'autre origine que les bavardages des nourrices :

"Toutes les fictions de ce genre, qui ne se proposent que de délecter l'auditoire, l'exposé philosophique les exclut de son sanctuaire pour les renvoyer aux berceaux des nourrices (in nutricum cunas) ${ }^{6}$. "

La figure de la nutrix, spécialiste des " contes de bonnes femmes ", sert à stigmatiser les auteurs de bas étage, ceux qui farcissent la tête de leur public de fariboles, de sornettes et de superstitions.

5. Farce nouvelle des Chamberières (entre 1480 et 1492), voir Recueil de farces françaises inédites du XVe siècle, publiées par Gustave COHEN, Cambridge (Mass.), Mediaeval Academy of America, 1949, p. 414, v. 75-93.

6. Macrobe, Commentaire au Songe de Scipion, I, 2, 9 (texte établi, trad. et commenté par Mireille Armisen-MarchetTI, Paris, Belles-Lettres, 2003). 


\section{Allaitement et soins aux nourrissons : un savoir partagé}

Cependant, on peut aller au-delà de ces lieux communs et de cette apparente mise à distance d'une communauté de femmes ignorantes, bornées dans leurs intérêts et leur vision du monde. Lorsque les textes s'arrêtent un tant soit peu sur des scènes impliquant le soin d'un nouveau-né, on s'aperçoit que les descriptions révèlent, malgré leur relative brièveté, une connaissance précise du métier de la nourrice. Elles comportent une énumération quasi formulaire des occupations de la nourrice, allaitement, bain, emmaillotage, coucher de l'enfant au berceau :

«"Fille, fet il, levez, levez!

Fu e chaundele m'alumez!

Un enfaunt ai ci aporté,

La fors el freisne l'ai trové.

De vostre leit le m'alaitiez;

Eschaufez lë e sil baigniez!"

Cele ad fet sun comandement :

Le feu alume, l'enfant prent,

Eschaufé l'ad et bien baigné,

Puis l'ad de sun leit aleité ${ }^{-7}$."

[" Ma fille, debout, levez-vous! Allumez-moi du feu et une chandelle, j’ai là un enfant que j'ai trouvé dehors, dans le frêne. Donnez-lui de votre lait, réchauffez-le et baignez-le! „ Elle s'est exécutée : elle a allumé le feu, elle a pris l'enfant, l'a réchauffé, baigné et lui a donné son lait.]

La distribution de ces différents soins est parfois répartie entre plusieurs nourrices, le plus souvent au nombre de trois :

"L'enfes fu nes; joie en font grant,

ét l'en li bailla maintenant

trois nouriches por lui servir,

por ennourir et por chierir.

L'une des trois l'avoit baignié,

et l'autre si l'avoit couchié;

la terche sert de l'alaitier

et de lui bien apparillier,

car, se Dex li donnoit croisture,

a tort perdroit par noureture ${ }^{8}$."

["L'enfant naquit : la joie fut grande et on lui attribua trois nourrices chargées de le servir, de le nourrir et de le chérir. L'une le baignait, la deuxième le couchait. L'office de la troisième était de l'allaiter et de l'habiller, car si Dieu lui accordait une bonne croissance, ce serait un tort de mal l'éduquer. "]

7. MARIE de FrAnCE, Lai de Fresne, dans Lais bretons (XII -XII ${ }^{e}$ siècles) : Marie de France et ses contemporains, éd. bilingue par Nathalie Koble et Mireille SÉGUY, Paris, Champion, 2011, v. 197-206, p. 280-283.

8. Le Roman des sept sages de Rome, édition bilingue des deux rédactions en vers français par Mary B. SPEER et Yasmina FoEHR-JANSSENS, Paris, Champion, 2017, v. 1185-1194, p. 142. Un passage semblable se trouve dans Jean RENART, L'Escoufle, éd. Franklin SwEETSER, Paris/Genève, Droz, 1974, v. 1788-1799. 
Au gré de ces réitérations, on perçoit que les soins aux nourrissons sont bien connus et répertoriés, même par les auteurs, en principe masculins, des romans. Les listes de soins de puériculture sont d'ailleurs tout à fait cohérentes avec les descriptions savantes qui recensent les tâches et les occupations des nourrices. La traduction par Jean Corbechon du Livre des propriétés des choses de Barthélémy l'Anglais nous en fournit un exemple éclairant :

" La nourrice est ainsi appelée parce qu'elle nourrit l'enfant à la place de sa mère $[. .$.$] Elle relève l'enfant quand il tombe, l'allaite quand il pleure,$ l'embrasse quand il se tait. Elle l'attache quand il remue trop, le lave et le nettoie quand il est sale ${ }^{9}$."

Ainsi, la belle indifférence aux tâches de la nourrice n'est-elle pas aussi complète qu'on veut bien le dire. Tant la littérature de fiction que les régimes de santé et les encyclopédies ${ }^{10}$ font apparaître une réelle préoccupation concernant la santé et la survie des jeunes enfants qui s'exprime bien au-delà du cercle restreint des nourrices.

Dans son Miroir de mariage, un texte foncièrement critique envers le mariage, comme les Quinze Joies de mariage, Eustache Deschamps fournit cependant une liste très détaillée des charges qu'implique la sollicitude envers les petits enfants. On y retrouve le principe énumératif qui gouverne les descriptions présentes dans les récits fictifs. Bien que cette attention de tous les instants soit prise en mauvaise part comme une peine (un " desconfort ") et un asservissement, le passage n'en témoigne pas moins d'une connaissance précise des gestes de la toilette, du nourrissage et des cajoleries que l'on réserve aux tout-petits :
"Enfans porter est desconfort,
Car il les faut enmailloler
Et tendrement enveloper,
Bercer, nettoier, conjouir,
Porter, chanter et resjouir,
Et leur ordonner blans drapeaulx,
Et les couvrir de douces peaulx,
De couvertoirs, de doulz liens,
Eulx couchier droit, faire tous biens,
Leur nombril estraindre et cerchier,
Et leurs cuissettes reverchier,
Faire papin ${ }^{11}$."

9. Le Livre des propriétés des choses. Une encyclopédie au XIVe siècle, mise en français moderne par Bernard RiBÉMONT, Paris, Stock, 1999, p. 143.

10. S'inscrivant dans la lignée des écrits antiques sur le sujet (Soranos d'Ephèse, Maladies des femmes et Galien), Aldebrandin de Sienne reprend des considérations bien connues des médecins. Sur les nourrices et la médecine antique, voir DASEN, Véronique, Le sourire d'Omphale : maternité et petite enfance dans l'Antiquité, Rennes, PUR, 2015, p. $249-280$.

11. Eustache Deschamps, CEuvres complètes, publ. par le marquis DE QuEUX DE SAINT-HILAIRE et Gaston Raynaud, Paris, F. Didot, 1878-1903, t. IX, Miroir de mariage, XXXIII, p. 101, v. 30093019 . 
[ "S'occuper d'un enfant est un embarras, car il faut les emmailloter et tendrement les envelopper, les bercer, les nettoyer, les cajoler, les porter, chanter des berceuses et les faire rire, leur préparer de beaux langes, les couvrir de douces pelisses, de couvertures et les attacher doucement, les coucher bien droit, leur administrer toutes sortes de soins, soigner et examiner leur nombril, scruter leurs petites cuisses, faire leur bouillie. "]

En faisant le compte des devoirs, dénoncés comme fastidieux, qui découlent de cette surveillance constante du nouveau-né, Eustache Deschamps dénombre aussi tous les aspects de la manipulation minutieuse du corps du nouveau-né. L'usage d'adverbes de manière (" tendrement ", v. 3011), d'adjectifs qualificatifs ("douces ", v. 3015; " doulx", v. 3016) et du diminutif (v. 3019) trahit la dimension affective et le souci de bien-être qui caractérisent cette sollicitude.

\section{Vulnérabilité}

Partagée par les hommes et les femmes, les nourrices et les lettrés, l'attention dévolue aux soins et à la nourriture qu'il faut procurer aux nourrissons repose évidemment sur la conscience très vive de la situation de vulnérabilité dans laquelle se trouve le nouveau-né dans un monde en proie à une mortalité infantile importante. C'est ici que les récits de fiction démontrent leur intérêt. Car cette préoccupation se traduit, surtout à partir du début du XIV ${ }^{\mathrm{e}}$ siècle, par la prolifération des récits d'expositions et d'enlèvements d'enfants qui sont autant d'expressions mythiques des dangers qui pèsent sur la progéniture humaine.

L'absence d'une nourrice est synonyme d'un danger de mort. Un récit exemplaire, enchâssé dans la totalité des nombreuses versions du Roman des sept sages de Rome, en témoigne tout particulièrement. Il s'agit d'un conte que la critique érudite identifie par un titre générique latin : Canis $^{12}$. Cette anecdote d'origine orientale figure dans le Panchatantra et est rapportée sous une forme différente par Pausanias ${ }^{13}$. Sous sa forme la plus répandue en Occident, elle raconte le dévouement d'un chien qui n'hésite pas à combattre au péril de sa vie un serpent. Le reptile s'est glissé près du berceau du fils premier-né de son maître, en profitant de l'incurie de ses nourrices (précisément celles dont les soins diligents sont pourtant répertoriés dans le passage cité plus haut), qui l'ont abandonné pour assister à un combat entre un ours et des mâtins ou à un tournoi. Les assauts du

12. Nous renvoyons ici à la plus ancienne version française connue de ce récit très répandu, datée du milieu du $\mathrm{XII}^{\mathrm{e}}$ siècle : Le Roman des sept sages de Rome, op. cit., v. 11651380. Sur ce récit, voir Fothr-JANSSENS, Yasmina, "Le chien, la femme et le petit enfant ", Vox romanica, 52, 1993, p. 147-163, et SPEER, Mary B., " The Prodigal Knight, the Hungry Mother and the Triple Murder: Mirrors and Marvels in the Dolopathos Dog Story ", dans Mullally, Evelyn, Thompson, John J. (éd.), The Court and Cultural Diversity, Selected Papers from the Eighth Triennal Congress of the ICLS, Cambridge, Brewer, 1997, p. 375-383.

13. Pausanias' Description of Greece, traduction James G. Frazer, Londres, Macmillan, 1898 , I, p. 551 ; IV, p. 421. 
serpent dévorateur révèlent les angoisses qui s'attachent à la question de la survie de l'enfant.

La vulnérabilité du moment de la naissance atteint son paroxysme dans des cas de naissances dans un espace sauvage, la mer ou la forêt notamment. Plusieurs romans et chansons de geste mettent ces périls en scène. Le Roman de Guillaume d'Angleterre en fournit un exemple qui date vraisemblablement de la fin du $\mathrm{XII}^{\mathrm{e}}$ siècle. La chanson de Beuve de Hantone, dont la plus ancienne version remonte probablement à la même période, comporte également une scène d'accouchement en milieu hostile. Ces deux récits s'inspirent de la légende chrétienne de Placide Eustache qui fournit un modèle aux très nombreuses œuvres médiévales basées sur le scénario de la famille dispersée. Beuve de Hantone et Guillaume d'Angleterre travaillent à rendre la séparation des membres de la famille plus pathétique en plaçant les circonstances de l'exil des parents avant la naissance des enfants, contrairement à ce que raconte la Vie de saint Eustache. L'enlèvement des enfants, par des bêtes sauvages dans le cas de Guillaume d'Angleterre, a alors lieu immédiatement après leur naissance. Au XIV ${ }^{\mathrm{e}}$ siècle, de très nombreux textes s'emparent de ce thème et le développent à loisir, en y adjoignant nombre de motifs merveilleux comme celui de la nourrice animale, sur le modèle de la légende de Romulus et Remus, ou celui de l'enlèvement d'un bébé par des êtres féeriques ${ }^{14}$. Les circonstances exceptionnelles qui entourent la naissance sauvage d'héritiers mâles contraignent parfois leurs pères à assumer les tâches "féminines " : emmailloter, baigner, endormir et nourrir l'enfant ${ }^{15}$.

Un passage du Roman de Guillaume d'Angleterre résume clairement les craintes que ne peut manquer de susciter la perspectives d'un accouchement sans nourrices ni " ventrières ". Guillaume, le roi d'Angleterre tente de dissuader son épouse enceinte de le suivre dans son exil :

"Ha! fait li rois, dame, merchi!

Par mon los vos remanrés chi,

Que trop estes grosse et pesans.

Por.C. mile mars de besans

Ne vauroie k'en ces boscages

M'avenist de vos nus damages.

Pres est l'heure, par tans venra,

14. De nombreuses chansons de geste dites tardives présentent un ou plusieurs épisodes de ce type. Citons notamment La Belle Hélène de Constatinople, Parise la duchesse, Lion de Bourges, Tristan de Nanteuil. Voir McCracken, Peggy, "The Wild Man and His Kin in Tristan de Nanteuil ", dans FABRY-TEHRANCHI, Irène, RuSSAKOFF, Anna (dir.), L'humain et l'animal dans la France médiévale (XII $-X V^{e}$ siècle), Amsterdam/New York, Rodopi, 2014; McCRACKen, Peggy, "Fantastic Lactations: Fiction and Kinship in the French Middle Ages " et Venturi, Céline, " Le lait du lion : identité épique et parenté mythique dans quelques récits profanes (XIII $-\mathrm{XV}^{\mathrm{e}}$ siècle)", dans ibidem, p. 37-43.

15. Voir FoeHR-JANSSENS, Yasmina, "Lignage et reproduction : aspects sociaux de la parenté et de la parentalité dans quelques chansons de geste ", à paraître dans les actes du colloque Aspects sociaux des littératures médiévales. Texte et situation communicative au Moyen Âge (5-8 septembre 2016, Moscou). 
Quë acoukier vos convenra

Et de vostre enfant delivrer.

A cui le porriés vos livrer,

A quels gardes, a quels nourices ${ }^{16}$ ?"

[" Ha, dit le roi, madame, de grâce! Vous resterez ici, comme je vous le conseille, car votre grossesse est très avancée. Même pour cent mille marcs de besants, je ne voudrais pas vous voir subir quelque malheur dans les bois. Le moment approche et arrivera bientôt où il vous faudra accoucher de votre enfant. À qui pourriez-vous le confier? À quels gardes? À quelles nourrices?"]

La vulnérabilité des nourrissons explique donc l'intérêt paternel pour les enjeux de la mise en nourrice, par ailleurs largement documenté ${ }^{17}$.

Quoique plus rarement, il arrive aussi que l'idée de la précarité de la vie enfantine affleure par un biais tout autre. Quelques mentions éparses viennent révéler la face obscure de la relation nourricière mercenaire : celle qui concerne le sort de l'enfant utérin de la nourrice. Le Roman de Merlin de Robert de Boron y fait une brève allusion, à propos de Keu, le sénéchal du roi Arthur. Ce personnage se caractérise dans toute la tradition arthurienne par son mauvais caractère et sa langue trop bien pendue. Selon Robert de Boron, il faut mettre cette acrimonie sur le compte du lait que Keu a tété comme enfant. En effet, il se nourrissait encore au sein de sa mère lorsque celle-ci s'est vu confier la garde du petit Arthur, fils nouveau-né du roi Uterpendragon et de la duchesse Ygerne. Relégué aux soins d'une nourrice de bas étage, Keu a été " desnaturez " pour avoir dû céder sa place à l'enfant providentiel dont Merlin a confié la garde à ses parents. Bien plus tard, le père de Keu plaidera la cause de son fils légitime auprès de son fils adoptif :

" [...] Et se il [Keu] est fols et vilains et fels, vos le devez bien soufrir, que ces mauvaises tesches a il eues por vos et prises en la garce que il alaita, et por vos norrir est il desnature $z^{18}$."

[ "S'il lui arrive d'être bizarre, malappris ou perfide, tolérez-le, puisqu'il a pris ces défauts dans le lait d'une femme de basse condition et que c'est pour que vous soyez nourri comme il se devait qu'il a perdu sa véritable nature ${ }^{19}$. "]

L'exemple de Keu fait ainsi apparaître l'envers du décor, le plus souvent inaperçu, et assez effrayant à vrai dire, de ce marché du lait. L'activité professionnelle de la nourrice cache la présence d'un enfant mis à l'écart au profit du nourrisson pour lequel ses parents se sont attaché les services de ladite nourrice. L'enfant utérin de cette dernière disparaît de la transaction,

16. ChRÉTIEN (?), Guillaume d'Angleterre, publication, traduction, présentation et notes par Christine FerLAMPIN-ACHER, Paris, Champion, 2007, v. 293-303.

17. Les travaux de Christiane KLAPISCH-ZUBER montrent que la recherche et l'engagement d'une nourrice à Florence est avant tout d'une affaire d'hommes, voir La maison et le nom : stratégies et rituels dans l'Italie de la Renaissance, Paris, Éditions de l'EHESS, 1990, coll. " Civilisations et sociétés ".

18. Robert DE Boron, Merlin, éd. Alexandre MichA, Genève, Droz, 1980, p. 278, §87, l. 19-24.

19. Robert DE BORON, Merlin : roman du XIII siècle, présenté, traduit et annoté par Alexandre MichA, Paris, GF-Flammarion, 1994, p. 167. 
il n'est qu'une ombre dans le tableau de l'allaitement mercenaire. On le voit parfois apparaître comme un souvenir distant qui n'a de consistance qu'au regard de la qualité du lait de sa mère. Les recommandations d'Eustache Deschamps à propos du choix de la nourrice nous fournissent un exemple de ces apparitions fugaces. La " connaissance du bon lait "implique la croyance en la qualité supérieure du lait de mâle, c'est-à-dire du lait d'une nourrice qui aurait accouché d'un garçon, mais l'existence de cet enfant est à peine évoquée. Seule compte son appartenance à un sexe ou l'autre, et encore cette donnée physiologique n'apparaît finalement que comme un attribut attaché aux qualités nourricières de sa génitrice :

" [...] et que l'en ait

La congnoissance du bon lait

Et du maintien de la nourrice,

Qu'elle ne soit sote ne nice,

Mais ait bon pis, soit lie et gaie,

Juene, jolie et se resgaie,

Que son lait sur l'ongle se [tiengne

Et ne soit vert, et ja n'aviengne

Que son lait ait un an passé,

Car l'enfant en seroit cassé,

Et en vaudroit pis durement;

Se masle a eu, certainement

Mieulx vault son lait que de femelle ${ }^{20}$."

[ «Il faut aussi avoir la connaissance du bon lait et celle du comportement de la nourrice : il faut qu'elle ne soit ni sotte, ni ignorante, qu'elle ait une bonne poitrine, qu'elle soit gaie et joyeuse, jeune, jolie et pleine d'entrain, que son lait tienne sur l'ongle et qu'il ne soit pas verdâtre, et il faut éviter que son lait ait plus d'un an, car l'enfant en serait gâté et il en subirait le préjudice. Si elle a eu un enfant mâle, son lait vaudra assurément mieux que s'il s'agit d'une femelle. "]

\section{Territoires de la fiction : Brun de la Montaigne et le lait de la fée}

\section{La nourrice "faée » : une demande paternelle d'excellence}

Naissances prodigieuses ou insolites, allaitements merveilleux par des nourrices animales, expositions et enlèvements, menaces de dévoration par des fauves ou des serpents, les fictions médiévales ne manquent pas de dramatiser les circonstances de la venue au monde des enfants humains dont elles entreprennent de narrer la geste épique ou l'itinéraire romanesque. L'examen d'un curieux texte, dont l'importance pour notre propos a été signalée récemment par Marie-Claire Gérard-Zai ${ }^{21}$, nous permettra de

20. Eustache Deschamps, EEuvres..., op. cit., Ix, Miroir de mariage, xxxIII, p. 101, v. 30193031.

21. GÉRARD-ZAI, Marie-Claire, " Nourrir les seins mercenaires au Moyen Âge ", dans DASEN, Véronique, GÉRARD-ZAI, Marie-Claire (dir.), Art de manger, art de vivre : nourriture et société 
démontrer que ces topiques narratives sont susceptibles de s'agencer en un ensemble cohérent qui en dit long sur l'imaginaire féodal de la reproduction. Brun de la Montagne ${ }^{22}$ place en effet la figure de la nourrice et la question de l'allaitement au centre des préoccupations lignagères et nous dévoile, du même coup, la force de leur potentiel fictionnel, qui prend ici une dimension quasiment mythique. Brun de la Montagne propose une véritable réélaboration littéraire des contenus de pensée qui construisent le " monde des nourrices ". Cette œuvre inachevée datée du XIve siècle se caractérise par des choix esthétiques singuliers dans la mesure où elle s'empare d'une matière narrative typique du roman d'inspiration bretonne ou arthurienne tout en empruntant sa forme (la laisse d'alexandrins rimés) au genre épique de la chanson de geste. Une telle tentative d'hybridation des deux principales formes narratives françaises n'a que peu retenu l'attention de la critique, en raison sans doute du caractère lacunaire du texte, qui ne nous est parvenu que dans un unique manuscrit, conservé à la Bibliothèque nationale de France sous la cote français 2170. Christine Ferlampin-Acher lui a toutefois consacré un intéressant article ${ }^{23}$. Ses conclusions nous incitent à considérer l'essai d'intégration des veines épique et romanesque médiévales comme un projet de revitalisation du récit arthurien " en utilisant une forme [la laisse épique] associée à la célébration d'un passé glorieux ${ }^{24}$ ». Le projet poétique de l'auteur reposerait donc sur un " parti pris nostalgique ${ }^{25}$ " conçu à une époque relativement tardive au cours de laquelle chacun des deux genres cherche à se réinventer. C. Ferlampin-Acher signale notamment la relative insistance avec laquelle la mélancolie des acteurs du récit est évoquée par le texte (v. 21, 901, 1147, 1242).

Tout l'intérêt de l'action se concentre au début du texte qui s'interrompt d'ailleurs après les tout premiers exploits du héros. Butor de la Montaigne, un seigneur déjà âgé, marié à une jeune et noble dame, vient d'avoir un fils, qui risque bien d'être l'unique héritier de son père. Pour assurer à cet enfant toutes les chances d'une vie heureuse et réussie, il forme le projet de l'exposer une nuit dans la forêt de Brocéliande au pied d'un arbre placé près d'une fontaine aux fées, afin que les habitantes de la source " destinent l'enfant ". Il espère que les dames de la fontaine lui accorderont des dons bénéfiques :

"Quant li sires le sot, Dieu prist a gracier

Quant il avoit .j. fil por s'onnour essaucier.

de l'Antiquité à nos jours, Gollion, Infolio, 2012, p. 87-102, les références à Brun de la Montaigne se trouvent p. 88 et 89, n. 2 et 6.

22. Brun de la Montaigne : roman d'aventure, publ. d'après le manuscrit unique de Paris par Paul Meyer, Paris, F. Didot, 1875.

23. Ferlampin-ACHER, Christine, "Brun de la Montagne : une chançon de matiere enforciee (v. 2744) ?", dans RÉGNIER-BoHLER, Danielle (dir.), Le Romanesque aux XIve et XVe siècles, Bordeaux, Presses de l'université de Bordeaux, 2009, p. 29-40; voir aussi eadem, Merveilles et topique merveilleuse dans les romans médiévaux, Paris, Champion, 2003, p. 330-332.

24. Ferlampin-Acher, Christine, "Brun de la Montagne... ", art. cité, p. 34.

25. Ibidem. 
Si pensa en son cuer, pour li plus avancier, Que porter l'en fera, sans plus de l'atargier, Delez une fontaine, assez près du rochier; Car il avoit repaire de fées ou gravier Qui aloient ou lieu touz dis esbanoier. Si ala tout errant huchier .j. messagier Et li a dit: "Amis, il te faut chevauchier Tost et viguereusement, ne veillez delaier. Va querre mes barons, car j'ai d'eus grant mestier, Et di qu'il veignent tost, por mon cors conseillier, Carj'ai .j. [moult] biau fil eü de ma mouillier Qu'as destinées veil tout errant envoier." " (v. 45-58)

["Quand le seigneur apprit [la nouvelle], il rendit grâce à Dieu puisqu'il avait un fils pour soutenir son honneur. Il forma en son cœur le projet de faire porter l'enfant, pour l'avantager, au bord d'une fontaine, près d'un rocher. Il y avait en effet sur le sable un lieu de rendez-vous des fées qui s'y rendaient tous les jours pour se divertir. Il appela aussitôt un messager et lui dit : "Amis, il te faut chevaucher immédiatement et à vive allure, ne tarde pas. Va quérir mes barons, car j'ai grand besoin d'eux. Dis-leur de venir bien vite pour me conseiller, car j'ai eu un beau fils de mon épouse, que je veux envoyer tout droit vers les fées." "]

Les vassaux du roi accueillent avec effroi cette perspective. Ils craignent que le nouveau-né ne succombe à l'épreuve d'une nuit passée dans la froidure de la forêt ou qu'une bête sauvage, ours (v. 294), lion ou serpent, ne s'empare de l'enfant et ne le dévore :

"Quant li mesages ot finée sa raison,

Ensemble sont alé tretout li cent baron

Et si ont dit entr'aus par fole entencion :

"Est nos sires meüs qui nous fait tel sermon

Qui se veult delivrer d'un si dous enfanson?

Il ne le pourroit miex metre a occission,

Car se li anfes est ostez de sa maison

Il en aura au cuer en brief temps marison

Car espoir trouvera ou serpant ou lyon

Qui l'anfant ara tost mis a destruction.” " (v. 84-93)

["Quand le messager eut terminé son discours, les cent barons se sont rassemblés et sans rien y comprendre, ils ont parlé entre eux : "Notre seigneur erre-t-il qui nous tient un tel raisonnement, veut-il se débarrasser d'un si doux bambin? Il ne pourrait pas mieux le mettre à mort, car si l'enfant est enlevé de la maison, il ressentira bientôt une grande douleur, car peut-être y aura-t-il un serpent ou un lion qui aura tôt fait de massacrer l'enfant." "]

Les délibérations entre Butor et ses vassaux se déroulent sur 600 vers environ. Elles se déploient au cours d'une scène d'ambassade, suivie d'un conseil des barons. Conformément à l'esthétique de la répétition dramatique propre à la chanson de geste, chaque étape du processus délibératif est l'occasion d'une nouvelle exposition du projet paternel et d'une réitération des doutes exprimés par les conseillers. La narration piétine, comme pour souligner l'importance et la gravité des décisions à prendre. Le rite propitia- 
toire n'a rien d'une affaire privée, c'est un acte politique dont il faut calculer soigneusement les risques. La mère de l'enfant n'est pas consultée. Une fois la décision prise, elle est mise devant le fait accompli et, tout en partageant les inquiétudes des dignitaires de la cour, elle se plie à la volonté de Butor (v. 554-625). L'emphase avec laquelle cette question est traitée permet de souligner le grave danger encouru par l'enfant, mais elle est peut-être aussi l'indice d'une distance critique, d'une intention comique de la part du narrateur qui jouerait du contraste entre un débat sur la croyance dans les fées et les graves questions stratégiques et militaires généralement discutées dans les scènes épiques de ce type.

Toujours est-il que Butor persiste dans son projet et charge quatre vassaux fidèles d'amener l'enfant à la fontaine. Finalement, tout se passe selon les vœux du père. L'enfant, soigneusement emmailloté dans des étoffes précieuses et chaudes, survit à son exposition et reçoit d'un trio de fées tous les dons dont un homme peut rêver : beauté, savoir et courage. Le cérémonial connaît cependant une légère perturbation, puisque la seconde des fées s'exprime en premier :
"La seconde, qu'en li ot bonnes voulentés,
Dit : "Dame, de par moi il sera estrinés,
Je li otroy qu'il ait en lui toutes biautés,
Et avec ce qu'i soit en tout si dotrinés
C'on puist dire partout qu'a bonne eure fu nés;
Et avec ce, qu'il soit en tous fais d'armes tés
Que de toute proesce il soit plus redoubtés
En guerres, en tournois, en tous fais esprovés,
Et de toutes gens soit plainement honnorés;
Car je croy bien qu'il fu de bon sanc engenrés.
Dame, faites li bien; grant ausmonne ferés

S'aucun bon don, pour Dieu, bonnement li donnés.

Se mes dons est petis, pour Dieu si l'amandés." " (laisse Lv, v. 955-968)

[" La seconde dame, pleine de bienveillance, dit : "Dames, de moi, il recevra des dons. Je lui octroie d'avoir en lui toutes les beautés et en plus, il sera très instruit, si bien qu'on pourra dire partout qu'il est né sous une bonne étoile. Et avec cela, qu'il soit, en tout fait d'armes, le plus redouté par sa prouesse, dans les guerres et les tournois, dans toutes les actions, et qu'il soit pleinement honoré de tous, car je crois bien qu'il a été engendré d'un bon sang. Dames, faites-lui du bien, vous agirez généreusement si vous lui donnez quelque beau don pour Dieu. Si mon don est petit, pour Dieu, améliorez-le." »]

Seul l'amour heureux est refusé à l'enfant par la première des fées, " la plus hautaine " (v. 942), outrée par l'entorse faite au protocole par la " moienne des trois" (laisse LVI, v. 968-983). Mais, pour contrecarrer ce don funeste, la troisième fée décide de devenir l'ange gardien de l'enfant, de s'attacher à lui et de l'aider dans toutes les circonstances de sa vie (laisse LVIII, v. 984-1003). On ramène l'enfant au château de son père, on le rend à sa mère, on le baptise et on lui donne le nom de Brun. La mère annonce alors n'avoir pas assez de lait pour nourrir son fils : 
"Ainsi que Butors fu entre lui et sa gent, Par dedens son chastel bel et courtoisement; Et que chascuns faissoit joie joieusement Pour l'enfant qui estoit nez tout nouvelement, Adont parla la dame a Butor doucement, Et dist : "Sire, pour Dieu, je ne sai vraiement Comment vos enfes puist estre nourris briement, Car je n'ai point de lait, sachiez certainement, Dont vivre se peüst, sans autre, longuement Si que je vous requier très amoureusement C'une nourice aions pour nourrir le cors gent De Brun de la Montaigne; or faites vistement Car besoing en a, selonc mon ensient." " (laisse cIv, v. 1860-1871)

["Alors que Butor tenait courtoisement son assemblée dans son château et que chacun se réjouissait de la naissance récente, la dame s'adressa doucement à lui : "Seigneur, au nom de Dieu, je ne sais vraiment pas comment votre enfant pourra être nourri dans peu de temps, car je n'ai pas de lait, sachez-le assurément, dont il puisse vivre, sans autre, longuement. Je vous demande donc très amoureusement que nous ayons une nourrice pour nourrir le noble corps de Brun de la Montagne. Faites vite, car il en a besoin, à ce que je sais." "]

C'est à ce moment-là qu'une femme apparaît, comme surgie de nulle part : il s'agit bien sûr, de la troisième fée, qui va devenir la nourrice de l'enfant. La beauté de la visiteuse providentielle fait resplendir les lieux de son apparition. Après les vœux des fées qui assouvissent les espérances du père en quête de grandeur pour sa progéniture, le don du lait féerique, dispensé par une femme " digne de tenir une grant roiauté " (v. 1947) épargne aux parents les inquiétudes liées à une toujours possible " dénaturation " de l'enfant par le lait de sa nourrice :

"Butor $[\ldots]$ a dit : $[\ldots]$

"Car nous avons nourrice a nostre voulenté

Pour nourir l'enfancon que vous avés porté."

La dame respondit: "Dites vous vérité?

- OyI, ce dit Butor, et par ma loiauté,

Onques ne vi plus belle en tretout mon aë

Vo chambre resplendit toute de sa biauté

Digne est bien de tenir une grant roiauté." " (v. 1935-1936 ; 1941-1947)

["Butor a dit [...] : "Nous avons une nourrice selon notre désir pour nourrir l'enfant que vous avez porté." La dame répondit. "Dites-vous vrai? — Oui, dit Butor, et ma foi, je n'en ai jamais vu plus belle de ma vie. Votre chambre resplendit entièrement de sa beauté, elle est bien digne d'être reine." "]

Sitôt laissée seule en présence de l'enfant, la fée, étrangement promue au rang de nourrice, procède à un curieux rite, que Laurence Harf-Lancner désigne comme un " baptême par le feu ${ }^{26}$ " et dont elle signale la présence dans deux autres textes, Les enfances Rénier et le Roman d'Aubéron. La

26. HARF-LANCNER, Laurence, « Le baptême par le feu : la survivance d'un rite dans trois textes épiques ", Senefiance, 21, 1987, p. 629-641. 
nourrice démaillote l'enfant et le chauffe dans le feu avant de le rhabiller soigneusement :

"Si tost que la dame ot desvelopé l'enfant,

Elle s'ala seoir delés .j. feu ardant,

Et de ses belles mains l'aloit souef portant,

Et derrière et devant moult doucement chaufant

Quant elle l'ot chaufé du tout a son commant,

Si le renvelopa en un plisson moult grant,

Et puis dont en un drap de bon fin or luissant. (v. 2005-2011)"

[ "Aussitôt que la dame eut démailloté l'enfant, elle alla s'asseoir à côté d'un feu ouvert et elle le maniait de ses belles mains, le chauffant très doucement par derrière et par devant. Quand elle l'eut entièrement chauffé à son gré, elle le renveloppa dans une grande pelisse, puis dans un grand drap d'or fin luisant. "]

S'appuyant sur un dossier comprenant des textes antiques, dont la Bibliothèque d'Apollodore commentée par J. G. Frazer, auquel elle ajoute le témoignage de Martianus Capella dans les Noces de Mercure et de Philologie et celui de plusieurs pénitentiels, L. Harf-Lancner montre qu'il s'agit là de la survivance d'une croyance en la possibilité d'assurer aux enfants, grâce au " pouvoir régénérateur du feu " une stature héroïque, voire l'immortalité ${ }^{27}$. L'apparition de ce rite coïncide souvent, comme dans le cas de Brun, avec la "substitution d'une mère surnaturelle à la mère mortelle ${ }^{28}$ ". On pourrait donc supposer que l'adoption des petits enfants par les fées, dont on connaît plusieurs exemples dans la littérature médiévale ${ }^{29}$, propose une organisation mythique alternative au fameux "mythe de la naissance du héros ", commenté par Otto Rank dans la foulée des travaux de Freud sur le complexe d'EEdipe. Si on en croit l'exemple de Brun de la Montaigne, cette construction mythographique portant sur l'allaitement surnaturel du héros relayerait un fantasme de grandeur qui s'appuierait non pas sur la conception de l'enfant par un père tout-puissant, mais sur le don de la première nourriture par une figure maternelle divinisée. L'ascendance glorieuse ne se constitue pas dans ce cas sur le mode de la conception, mais sur celui de l'adoption : la parenté féerique est toujours déjà marquée par l'élection et la fiction.

Cette curieuse fée nourrice n'est pas tout à fait isolée dans la littérature médiévale. Le témoignage de Jean d'Arras, l'auteur, au début du $\mathrm{xv}^{\mathrm{e}}$ siècle, d'un Roman de Mélusine, est sollicité par L. Harf-Lancner à propos du rite de cuisson magique des enfants. Or la fée tutélaire du lignage des Lusignan n'est pas sans cultiver quelques liens avec l'univers de la maternité. Elle

27. HARF-LANCNER, Laurence, " Le baptême par le feu... ", art. cité, p. 636-638.

28. L'une des descriptions des soins donnés aux petits enfants citées ci-dessus ajoute à la liste canonique des gestes de puériculture, le fait de réchauffer l'enfant. Il s'agit de la citation du Lai de Fresne. Le contexte de cette scène est justement celui de l'adoption d'un enfant exposé.

29. Ce scénario apparaît dans Floriant et Florete, le Lancelot en prose, le Roman de Cassidorus, Maugis d'Aigremont ou Perceforest. 
appartient au groupe assez restreint de fées qui donnent le jour à des enfants. Mélusine est fée et mère; à la fin du récit tel qu'il est rapporté dans la version en vers de Coudrette, elle prend elle-même à sa charge, malgré la malédiction qui la tient désormais éloignée de Lusignan, l'allaitement de ses plus jeunes fils. Le texte précise d'ailleurs qu'elle " chauffoit, alaistoit, recouchoit " ses fils ${ }^{30}$. Plusieurs héros arthuriens sont, quant à eux, enlevés par des fées qui prennent en charge leur éducation. Le plus connu d'entre eux est Lancelot, ravi tout petit par la fée Viviane, la Dame du Lac, au moment du départ en exil de ses parents et de la mort de son père. Le Roman de Lancelot en prose n'affirme pas explicitement que Viviane est la nourrice de l'enfant qu'elle recueille, mais le récit des circonstances du rapt du nourrisson fournit un indice ténu qui évoque l'allaitement. Au moment de décrire le geste par lequel la " demoiselle " s'empare de l'enfant pour le tenir contre elle, le narrateur mentionne explicitement les seins de la fée entre lesquels elle serre le nourrisson :

"Et qant ele [= la mère de Lancelot] aproche des chevax qui estoient desus lo lac, si voit son fil hors del bercuel tot deslié, et voit une damoisele qui lo tenoit tot nu en son giron et l'estraint et serre moult doucement entre ses mameles et li baise les iaux et la bouche menuement ${ }^{31}$."

[Quand elle est près des chevaux, qui étaient restés au bord du lac, elle voit son fils hors de son berceau et dévêtu; elle voit une demoiselle qui le tient tout nu dans son giron et l'étreint et le serre doucement contre sa poitrine [entre ses seins] et lui baise les yeux et la bouche inlassablement].

Brun de la Montagne, en conjoignant deux formes de distinctions héroïques dévolues aux nouveau-nés, le vœu des fées et le baptême de feu, et en rendant explicite la figure, ébauchée ailleurs, d'une fée nourrice, pointe l'outrance des exigences paternelles à l'égard de la nouvelle génération chevaleresque. Sommées de combler ce désir d'accomplissement héroïque que le seigneur sur le déclin nourrit à l'égard de son fils, les fées deviennent les garantes de cette aspiration incommensurable. L'adoption de l'enfant par une figure maternelle d'essence merveilleuse, protectrice et nourricière semble de nature à calmer les angoisses paternelles.

\section{La mélancolie des « seigneurs d'ancesserie » : féérie et superstitions masculines}

Par-delà cette première conclusion, le montage narratif de cette curieuse entrée en matière mérite une analyse plus détaillée. Il rassemble en effet en un récit continu le souvenir de plusieurs motifs narratifs distincts, pour en tirer un récit original.

30. Coudrette, Le Roman de Mélusine ou Histoire de Lusignan, éd. Eleanor RoAch, Paris, Klincksieck, 1982, v. 4380.

31. Lancelot du Lac, éd. Elspeth KenNEDY, trad. François Mosès, Paris, Le Livre de Poche, 1991, Chapitre III, p. 76-77. 
Quiconque connaît les Lais de Marie de France ${ }^{32}$ ou le Roman de Mélusine ${ }^{33}$ aura reconnu dans la fontaine aux fées, le lieu topique où de jeunes héros à peine sortis de l'adolescence rencontrent la merveilleuse fée amante qui pourvoira à leur bonheur, en leur offrant l'amour en même temps que l'aisance matérielle. Par ailleurs, la scène des " étrennes ${ }^{34}$ " offertes par les fées à titre d'heureux présage fait directement référence aux figures de fées marraines, fréquentes dans les contes de fées, mais aussi dans plusieurs fictions narratives comme Ogier le Danois, Parise la duchesse, Artus de Bretagne ou encore Perceforest. Brun de la Montagne opère la jonction entre la tradition des fées amantes et celles des fées marraines dont L. Harf-Lancner a étudié la généalogie ${ }^{35}$. Si les fées marraines sont sans conteste les héritières des Parques, la dénomination qu'elles partagent avec les créatures des eaux et des forêts, ces sylvaticae ${ }^{36}$ descendantes des nymphes et/ou de divinités celtiques, atteste de leur commune inféodation aux puissances de la destinée. Les fées sont les fata, les destinées, qu'elles règnent sur la petite enfance des hommes ou qu'elles affirment leur empire au moment de l'éveil de la sexualité.

L. Harf Lancner se sert de l'exemple de Brun de la Montagne pour démontrer l'absence de solution de continuité qui existe dans la littérature médiévale entre les deux types folkloriques de la fée amante et de la fée marraine ${ }^{37}$. Les fées marraines de Brun ont toutes les séductions des amantes merveilleuses évoquées par les lais féériques : corps blancs de neige, riches atours, vêtements étroitement lacés laissant apparaître la chair désirable :

"Les dames dont je di si estoient faées

Qui si très noblement estoient asesmées.

Leur cors furent plus blanc que n'est noif sor gelée,

Et si très chiérement estoient atournées,

Car de couronnes d'or furent toutes dorées

Et de blans dras de soie estoient aournées

Enmi de la poitrine estoient escollées. " (v. 923-929)

[ "Les dames dont je parle étaient des fées qui étaient vêtues très noblement. Leurs corps étaient plus blancs que n'est la neige sur la glace et elles étaient richement parées, car des couronnes d'or les enluminaient et elles portaient maints manteaux de soie. Leurs poitrines étaient décolletées. "]

32. Marie de France et ses contemporains, éd. bilingue par Nathalie KoBLE et Mireille SÉGUY, Paris, Champion, 2011.

33. Jean D’ARRAS, Mélusine ou la Noble Histoire de Lusignan, éd. Jean-Jacques VINCENSINI, Paris, Librairie générale française, 2003; COUDRETTE, Le Roman de Mélusine ou Histoire de Lusignan..., éd. Eleanor ROACH, op. cit.

34. Tel est le terme qui sert à désigner les dons des fées : estriner v. 956, 1115, 1659, 3638.

35. HARF-LANCNER, Laurence, Les fées au Moyen Âge : Morgane et Mélusine, la naissance des fées, Paris, Champion, 1984.

36. Le terme apparaît, pour désigner les fées agrestes, dans le pénitentiel de BURCHARD DE Worms (Decretum, P. L. CXL, col. 971). Le passage est cité et traduit par HARF-LANCNER, Laurence, Les fées au Moyen Âge..., op. cit., p. 23-24.

37. HARF-LANCNER, Laurence, Les fées au Moyen Âge..., op. cit., p. 42-46. 
La mention du décolleté des fées est particulièrement intéressante : elle assure la jonction entre fonction nourricière et attrait érotique des fées et prépare l'émergence de la fée nourrice.

À la lumière de cette intervention du merveilleux féerique dans les rites propitiatoires entourant la naissance, il est tentant de reconsidérer la présence récurrente de trois (parfois quatre) nourrices auprès des enfants longtemps désirés que nous avons signalée plus haut. On se demandera si ce trio de femmes n'est pas à mettre en relation avec les Parques ou leurs représentantes médiévales, les fées. Dans ses travaux sur la petite enfance dans les mondes grec et latin, Véronique Dasen propose un rapprochement de même type entre les sages-femmes et la triade des Parques et signale la "dimension bienveillante " et nourricière que prennent ces déesses dans la Gaule romaine ${ }^{38}$. Lorsqu'elles apparaissent comme actrices dans le récit, comme dans le récit Canis du Roman des sept sages de Rome, les femmes en charge de la survie de l'enfant tissent par leurs soins, ou leur négligence, quelque chose du destin de l'enfant.

Mais le texte entrelace également le scénario du vœu des fées avec celui de l'exposition de l'enfant. Comme le rappelle L. Harf-Lancner, la visite des fées marraines, attendue lors de la naissance, est sollicitée au moyen d'un rite domestique : on dresse une table afin d'assurer aux fées le meilleur accueil possible :

"À la naissance d'un enfant, on a coutume, dans les maisons bien tenues, de dresser une table à l'intention des fées marraines qui viendront peut-être rendre visite à l'accouchée et, surtout, décideront du destin du nouveau-né ${ }^{39}$. "

À la faveur du changement de décor qu'il impose à la cérémonie des " étrennes " des fées, le projet de Butor de la Montagne évoque, outre la rencontre avec la fée amante, un rite qui consiste à déposer un enfant en dessous ou au creux d'un arbre. En souhaitant interroger les " destinées " de l'enfant, le père répète les gestes typiques de l'abandon d'enfants, dans les récits mythologiques d'exposition, mais aussi dans certains récits médiévaux comme le Lai de Fresne. Il reconduit en outre une pratique plus ou moins funèbre qui fait intervenir la croyance aux changelins. Les enfants chétifs et maladifs, qui ne prospèrent pas, que le lait maternel ou mercenaire ne fait pas grandir, sont réputés avoir été substitués nuitamment à un enfant humain bien portant par des êtres merveilleux : ce sont des changelins ${ }^{40}$. Leurs mères vont les déposer au bord d'un point d'eau, parfois elles les y baignent. Par ce rite, elles se proposent de rendre le nourrisson souffreteux à ses parents non humains dans l'espoir, d'avance déçu, de retrouver le leur en bonne santé. C'est une manière de gérer le deuil et la perte d'un enfant : le nourrisson affaibli n'est pas le nôtre, l'enfant véritable vit heureux parmi les fées. Le récit Canis, déjà commenté ici, se trouve asso-

38. DASEN, Véronique, Le sourire d'Omphale..., op. cit., 2015, p. 223-247 (chapitre VIII, « Les Parques et le pouvoir des femmes").

39. HARF-LANCNER, Laurence, "Le baptême par le feu : la survivance... ", art. cité, p. 629.

40. Doulet, Jean-Michel, Quand les démons enlevaient les enfants : les changelins, étude d'une figure mythique, Paris, Presses de l'université de Paris-Sorbonne (PUPS), 2003. 
cié à un rite de ce type dans la somme théologique d'Étienne de Bourbon, dominicain du XIII ${ }^{\mathrm{e}}$ siècle ${ }^{41}$. Le savant prédicateur et inquisiteur raconte comment, lors d'une de ses tournées dans la région des Dombes, il a appris l'existence d'un saint dont il ignorait jusque-là l'existence, saint Guinefort. Menant l'enquête un peu plus avant, il apprend avec stupéfaction que ce saint inédit n'est autre qu'un chien dont la légende rapporte le dévouement héroïque du lévrier fidèle pour le salut de l'enfant de son maître que l'on peut lire dans le Roman des Sept Sages. Le lieu de culte de saint Guinefort est le théâtre d'expositions de changelins. Jean-Claude Schmitt a consacré une étude célèbre à ce dossier, au début de laquelle il reproduit et traduit le texte de l'inquisiteur qui n'hésite pas à désigner l'exposition des enfants comme une pratique criminelle et à traiter les femmes qui s'y livrent de "mères infanticides" (matricidae) ${ }^{42}$.

La similarité entre les agissements des paysannes des Dombes et ceux du seigneur de la Montagne est suffisamment grande pour que l'on puisse distinguer, sous la volonté de doter l'enfant des meilleures chances dans la vie, une prise de risque qui dévoile les craintes parentales, plus particulièrement dans le cas de Brun de la Montagne, les craintes paternelles concernant la survie de l'enfant. On sait que les reproches des vassaux fidèles ne manquent pas de rappeler les périls inhérents à ce projet. On concevra donc combien le projet de Butor à l'égard de son fils est gros de contradictions : il veut s'assurer le meilleur pour son enfant, mais n'hésite pas à l'exposer à la mort pour cela. La demande d'excellence adressée à l'héritier unique prend un tour inquiétant : "Sois le meilleur, ou meurs. "

C'est peut-être précisément le caractère profondément ambivalent de cette exposition des enfants nouveau-nés, que désigne le terme de " mélancolie " dont C. Ferlampin-Acher a repéré l'usage répété dans Brun de la Montagne et qu'elle interprète comme l'expression d'une volonté de restauration de la tradition chevaleresque. Les quatre occurrences de ce terme savant se trouvent en effet tous dans des passages qui ont à voir avec les vœux des fées. Dès la première laisse, on le voit apparaître, au v. 21, pour désigner l'état d'esprit dans lequel « les rois, ducs et princes faisaient porter leurs enfants en forêt " :

"Vous aves bien ouï qu'en vielle ancesserie

Que roy et duc et prince, ou honneur est florie,

Quant ooient parler d'aucune faierie,

Pour ce que destinée en leur hoirs fust norrie,

Faissoient leur enfans porter par leur meisnie

En forès et en bois par leur melancolie,

Ou fust en haut desert ou dedens praarie,

Desous arbre ramu ou fontaingne jolie,

41. Étienne de BourBon, Traité des sept dons du saint esprit, voir A. LeCOY DE LA MARCHE, Anecdotes historiques, légendes et apologues tirés du recueil inédit d'Étienne de Bourbon, dominicain du XIII siècle, Paris, 1886.

42. Sснмітт, Jean-Claude, Le saint lévrier : Guinefort, guérisseur d'enfants depuis le XIII siècle, Paris, Flammarion, 1979, p. 14 pour le texte latin, p. 16 pour la traduction. 
Afin que leur enfant amandassent leur vie

Par destinée qui fust a aus otroïe. " (v. 16-25)

["Vous avez bien entendu raconter que, dans les temps anciens, les rois, les ducs et les princes dans lesquels l'honneur s'illustre, lorsqu'ils entendaient parler de quelque féérie, afin d'assurer la destinée de leurs héritiers, faisaient porter leurs enfants par leurs familiers en forêt et dans les bois, sous l'impulsion de leur désir mélancolique, que ce soit dans des lieux très isolés ou dans des prairies, sous les arbres feuillus ou près d'une belle source, afin que la vie de leurs enfants soit rendue meilleure par une destinée [heureuse] qui pourrait leur être octroyée. "]

Cette " mélancolie " s'apparente à une forme de pensée obsédante ${ }^{43}$ qui caractérise les nobles "de vielle ancesserie ", obnubilés par la nécessité d'assurer la destinée de leurs héritiers et par leur volonté d'alimenter ("nourrir ") sans cesse leur rêve de perfectionnement ou d' " amendement " héroïque. Toujours est-il que la mort possible de l'enfant hante le désir d'un destin hors norme. Le meilleur des vassaux de Butor, Bruiant, figure de père plus humain que celle du géniteur, ne cesse de dénoncer l'hybris de ce dernier et cherche à s'opposer à son projet virtuellement infanticide. À la faveur des multiples réitérations qui scandent cet épisode, un débat s'instaure sur la pertinence du rite d'exposition et sur la valeur de cette croyance superstitieuse. Cette manière de discuter le substrat folklorique de la matière narrative est caractéristique du genre romanesque, selon C. Ferlampin-Acher. Le merveilleux n'y est pas un objet de croyances indiscutables. Il fournit matière à controverses et à de multiples interprétations contradictoires ${ }^{44}$. Ainsi le messager envoyé par Butor auprès de ses vassaux rapporte-t-il à son maître les objections de ces derniers qui ne semblent pas loin de penser que leur vieux seigneur " radote " (v. 110, $507)$ : «Et ils m'ont respondu [...]/que scïance est en vous plainement radotée ", v. 291.

Sous la plume du dominicain Étienne de Bourbon, les superstitions sont tout uniment le fait d'un imaginaire féminin dévoyé. La croyance aux changelins et aux vertus curatives de la fontaine et de l'arbre dédiés au chien martyr offre un exemple parfait des inepties répandues par de «misérables

43. Le terme " mélancolie " d'origine savante et médicale désigne en ancien français divers états mentaux comme des manies ou des lubies, voir ToBLER, Adolf, LOMMATZSCH, Erhard Friedrich, Altfranzösisches Wörterbuch, t. 5, Wiesbaden, 1960, 1353, 5-1356, 8. Dans un passage du Roman de Perceforest, il apparaît dans un contexte lié à la procréation. En accord avec la croyance selon laquelle l'activité imaginaire de la mère peut influer sur la conformation de l'enfant qu'elle porte, une fée, mère d'un enfant velu comme un ours, déclare : "Telle fut l'aventure que l'enffant vint sur terre pelu comme ung ours par la melancolie que j'avoie, en le concevant, sur Estonné que j'avoie mué en ours. " (Perceforest, L. IV, p. 1002, cité par FERLAMPIN-ACHER, Christine, Fées, bestes et luitons : croyances et merveilles dans les romans français en prose [XIII -XIV siècles], Paris, Presses universitaires de Paris-Sorbonne, 2002, p. 286).

44. FERLAMPIN-ACHER, Christine, Merveilles et topique merveilleuse dans les romans médiévaux, Paris, Champion, 2003, p. 171-266, sur Brun de la Montagne, voir p. 330-332; eadem, "Chançon [...] esforciee... ", art. cité, p. 33-34. 
jeteuses de sort" (misere mulieres sortilege $)^{45}$. Ce sont encore des femmes, des mères et des vieilles qui perpétuent le culte du saint animal. Le curieux " roman épique " de Brun de la Montagne, tout au contraire, plutôt que de reprendre, à propos des fées, le stéréotype des contes de nourrice, installe la croyance aux fées au centre d'un débat entre hommes ${ }^{46}$. Il en fait la pierre de touche d'un imaginaire masculin de la noblesse porté par une tradition narrative qui revendique son ancienneté, au risque de la redite, et qui affronte délibérément la contestation.

\section{Un enfant pour un autre : du changelin au fils fortuné}

Il est bien difficile de démêler qui, de Butor ou de Bruiant, l'emporte dans ce débat. Certes, le père obtient ce qu'il veut et assure à son fils, grâce au lait de la nourrice faée, la destinée glorieuse à laquelle il se doit de prétendre. Cependant les réticences et les préventions de Bruiant ne restent pas à l'état de suppositions; elles trouvent leur confirmation narrative au cours de la mission que lui confie son seigneur : veiller sur l'enfant durant le déroulement du rite féerique. Or sur le chemin qui le conduit à la forêt de Brocéliande, il entend soudain les lamentations d'une femme; s'approchant de la source des cris, il découvre une épouse éplorée tenant enlacé le corps de son mari. Cette scène est bien connue des lecteurs de romans bretons. Le plus souvent, un tel détour aventureux ouvre sur un épisode adventice qui offre au héros l'occasion de venger glorieusement la mort du mari ou de l'ami défunt, victime d'adversaires félons, de géants cruels ou de chevaliers dévoyés. Mais, dans ce cas, le deuil de la femme sert de relance à l'aventure principale. La dame confie en effet à son interlocuteur qu'elle a aussi perdu son enfant nouveau-né en l'exposant à la fontaine aux fées. Son double deuil rend donc inutile toute tentative de lui venir en aide :

"La dame li respont: "Vo paine perdriez,

Si vous requier merci que vous vous en ailliés,

Sur le cors monseigneur demanter me laissiés.

[...]

Sire ce dit la dame, or ne vous merveilliés,

Quant je pers mon seigneur se mes cuers est corciés,

Car avecques ce m'est mes anfes chalangiés,

Qui pour eür avoir estoit ci adreciez."

XLIII

Et quant Bruians oy la dame ainssi parle[r],

Si li vot doucement enquerre et demander

A quel fin la avoit son enfant fait porter.

La dame li respont: "Pour honneur conquester,

Pour avoir destinée et pour avanturer."

"Dame, ce dit Bruians, le pourroit on trouver?"

45. Sснмітт, Jean-Claude, Le saint lévrier..., op. cit., p. 13 et 15.

46. L'étude de Jean-Michel Doulet, Quand les démons enlevaient les enfants..., op. cit., sur les changelins place au centre de la réflexion un trio de personnages formé par la fée, la mère et l'enfant. 
La dame respondi: "La mort l'a fait finer,

Car.I. crueus serpant si le vint estrangler." "(v. 771-789)

[" La dame lui répond : "Ce serait peine perdue, c'est pourquoi je vous demande de vous en aller, sur le corps de mon mari, laissez-moi me lamenter. $[\ldots]$

Seigneur, dit la dame, ne soyez pas étonné si mon cœur est plein de chagrin en perdant mon mari, car en plus mon enfant m'a été enlevé, lui qui, en vue de sa bonne fortune, avait été envoyé ici."

Quand Bruiant entend la dame parler ainsi, il souhaite demander avec tact à quelle fin elle avait fait porter là son enfant. La dame lui répond : "Pour acquérir de l'honneur, pour recevoir les dons des fées et pour le mettre en aventure."

"Dame, dit Bruiant, pourrait-on le retrouver?" La dame répondit : "La mort l'a mené à sa destruction, car un serpent cruel est venu l'égorger ${ }^{47}$." "]

L'évocation de la mort du nourrisson tué par un serpent a pour vocation d'avérer les craintes de Bruiant et de donner à voir les dangers de l'exposition prévue par un père qui ne recule pas devant la perspective d'un infanticide.

Bien plus, la suite du récit confirme l'importance de la présence fantomatique de l'enfant mort, "étranglé par le serpent ". Si l'on s'interroge pour savoir d'où vient le lait de la fée nourrice qui se présente au château, on se souviendra de cette rencontre : la mère éplorée serait-elle un avatar de la fée nourricière? La fée prétend en effet avoir récemment perdu son mari et un enfant :

"Et si tost qu'elle fu en la salle votie,

Elle dit a Butor: "Sire, mes cuers vous prie

Que vous me retenés avecques vo meisnie,

Car je sui gentilz fame et de haute lignie.

Si sui de mon païs, n'a pas trois jors, partie;

Or est mors mon seigneur, du cors osté la vie;

Et s'avoie .j. enfant ausi qui ne vit mie,

Lequel je nourrissoie et avoie en baillie.

Si sui venue a vous, car on me certefie

Que vo mouillier ajut hier, en la nuit serie." "(v. 1905 -1915)

["Et aussitôt qu'elle fut dans la salle voûtée, elle a dit à Butor : "Seigneur, je vous prie de tout mon cœur de me retenir dans votre maison, car je suis une femme noble et de haute lignée. J'ai quitté mon pays il n'y a pas trois jours. Mon mari est mort à présent, il a perdu la vie et j'avais aussi un enfant qui ne vit plus, que je nourrissais et dont je m'occupais, je suis venue à vous, car on me certifie que votre épouse a accouché hier au cours de la nuit." "]

Il faut avoir enfanté pour être une nourrice : comme dans le cas de Keu, il y a toujours un enfant mort, sacrifié ou du moins tenu à l'écart, dans les coulisses d'un allaitement mercenaire. Cet enfant perdu est comme le double funèbre ou tragique de l'enfant vivant. Lorsque Bruiant revient de la

47. Je traduis par " égorger " plutôt que " étrangler " car au v. 1138, ce verbe est utilisé à propos du danger que la présence d'un lion pourrait faire courir à l'enfant. 
fontaine aux fées, il chevauche en tenant dans ses bras son jeune seigneur. Au fil de la chevauchée, le souvenir de la dame éplorée lui revient et cette pensée fait ressurgir le motif de la mélancolie (v. 1147). Mais la présence de l'enfant bien vivant lui fait oublier ses sombres pensées : l'image de l'enfant sacrifié s'efface au profit de son possible frère de lait :

"Adont s'en sont venus bellement chevauchant,

Por ce que li petis enfes aloit dormant;

Enmi de la forest s'en vindrent tout parlant;

Mais Bruians s'en aloit tout melancoliant,

Car il li souvenoit de la dame plaissant

Qui son seingneur ot mort, qui faissoit duel si grant,

Onques dame ne fist plus grant en son vivant;

Mais nonpourquant Bruians l'ala tout oubliant

Pour l'enfant qu'il tenoit entre ses bras devant.

Ainsi ont trespassé le bois de Bersillant. " (v. 1144-1153)

[ "Ils s'en venaient en chevauchant paisiblement, parce que le petit enfant dormait. Ils sont arrivés au milieu de la forêt tout en parlant. Mais Bruiant avançait pris dans des pensées mélancoliques, car il se souvenait de la plaisante dame dont le mari était mort et qui manifestait un chagrin si grand que jamais dame n'en avait connu de pareil. Mais pourtant Bruiant finit par l'oublier à cause de l'enfant qu'il tenait dans ses bras. C'est ainsi qu'ils ont traversé la forêt de Brocéliande. "]

Inachevé, peut-être parce qu'inachevable, Brun de la Montaigne s'avère pourtant une œuvre d'un grand intérêt. En thématisant les angoisses à propos de la mort prématurée des enfants et les attentes immodérées des pères à l'égard de leurs fils, elle promeut explicitement un personnage merveilleux que d'autres textes se contentent d'évoquer : la fée nourrice est conviée à répondre aux inquiétudes féodales autour de la reproduction. L'exposition initiale du fils trop aimé, obtenue par la superposition de plusieurs motifs merveilleux différents, a ceci d'intéressant qu'elle recycle toute une série de croyances (présence des fées marraines à la naissance d'un enfant, fontaines hantées par des fées amantes, substitutions maléfiques d'enfants) qui relèvent nettement de superstitions dénoncées par l'Église et régulièrement disqualifiées comme des enfantillages colportés par d'ignorantes nourrices. Loin de s'en tenir à cette facilité, le roman épique s'empare de la plurivocité du schéma mythique, pour l'installer au centre d'un débat entre hommes. La croyance aux changelins change de genre, elle cherche à accueillir et apaiser une angoisse masculine. Contrairement à la variante paysanne et féminine décriée par Étienne de Bourbon, cette version littéraire du mythe de l'enfant échangé ne vient pas recouvrir le deuil d'un enfant malade, mais permet au contraire de tenir à distance la possible débilité du fils en retournant le signe qui s'attache au rejeton des fées. Ici le changelin est promis à la gloire.

Pourtant, à chaque étape de ce récit de toute petite enfance, la présence conjointe du risque aventureux de la vie et du péril de la mort délivre une leçon poignante sur le projet paternel en contexte chevaleresque : l'édu- 
cation du futur chevalier voue d'emblée l'enfant à la mort ce qui suscite la mélancolie ${ }^{48}$ des pères. Butor veut le meilleur pour son fils, mais il sait qu'à chaque moment, ce souhait l'expose au pire. La mort du fils est inscrite au creux de la destinée chevaleresque, tant et si bien que l'enfant exposé à la fontaine aux fées est toujours, à l'image de son double disparu, un revenant.

Apparemment de peu de conséquence et purement anecdotique, la représentation de l'allaitement des petits enfants n'a jamais vraiment retenu l'attention des chercheurs et chercheuses en littérature médiévale. Pourtant lorsque l'on s'y attache, il s'avère que le don du lait revêt dans les fictions médiévales une signification symbolique forte. Bien que les textes ne soient pas exempts de préjugés négatifs sur les nourrices, la nourriture des tout petits, le plus souvent des enfants mâles, constitue en effet une donnée importante de la fabrique des héros. Il arrive que le motif prenne de l'ampleur et s'allie à des représentations empreintes de merveilleux comme la présence de nourrices de féerie ou d'animaux providentiels offrant leur lait à un enfant exposé. Le lait des fées qualifie le futur chevalier et fournit les prémices de sa gloire, mais le remplacement de la figure maternelle par une instance surnaturelle est aussi l'occasion de rappeler les dangers qui menacent la vie du nourrisson. La mort rôde autour des berceaux : le lait et les soins d'une créature merveilleuse ont pour fonction de rappeler et de signaler ce péril tout en l'exorcisant. Les scènes d'allaitement s'inscrivent ainsi dans une topique bien établie et témoignent d'un intérêt indéniable pour les modes d'éducation (" nourreture ", en ancien français) qui gouvernent la construction des carrières héroïques. Elles relayent également les inquiétudes masculines à l'égard de la progéniture humaine. En mobilisant la féerie dès les premiers instants de la vie de leurs héros, les romans et les chansons de geste constituent donc une sorte de mythographie de la naissance et de la petite enfance dont la polyphonie demande à être examinée dans toute sa complexité, puisqu'elle nous incite d'une part à repenser le primat de la seule puissance procréatrice du père dans la production de la gloire des fils et d'autre part à prendre en compte la part d'ombre et de doutes qui s'attache à ces trajectoires héroïques.

\footnotetext{
48. Tant la définition moderne de la mélancolie, comprise depuis les travaux de Freud comme un deuil interminable, que la connivence établie dès le Moyen Âge entre l'humeur noire et le péché d'acédie ou de désespérance, se révèlent probantes pour décrire la conscience douloureuse du caractère tragique de toute éducation chevaleresque qui hante Butor de la Montagne.
} 


\section{RÉSUMÉ}

Élaboré dans le cadre du programme de recherche "Lactation in history " financé par le fonds national de la recherche scientifique suisse (FNRS) et consacré aux représentations et aux pratiques de l'allaitement dans la longue durée, le présent article se propose de considérer les soins prodigués aux nourrissons sous l'angle de leur représentation fictionnelle dans la littérature française du Moyen Âge. Ce parti pris permet de montrer que, contrairement à un certain nombre d'idées reçues, les grands genres narratifs médiévaux accordent une attention soutenue aux faits et gestes qui entourent la venue au monde des humains. Les mentions des rites et des pratiques liés à la naissance et à la petite enfance offrent un panorama imaginaire très précis concernant les enjeux de la reproduction humaine, dans sa composante sociale, notamment. La fabrique du héros prend, en particulier au cours du $\mathrm{XIII}^{\mathrm{e}}$ siècle et au $\mathrm{XIV}^{\mathrm{e}}$ siècle, un tour particulier. Tout un appareil de motifs merveilleux, d'origine folklorique aussi bien qu'hagiographique, vient s'attacher à la description des soins et de l'accueil prodigués aux nourrissons, leur conférant une aura féerique qui confine à la mise en place d'une sorte de mythologie aux contours certes indécis, mais dominée par l'action de puissantes figures nourricières dont il convient de déterminer la fonction, qui peut varier d'un texte à l'autre.

\section{ABSTRACT}

This article, prepared within the interdisciplinary research team "Lactation in history" financed by the Swiss National Science foundation, focuses on the representation of the care given to new born children as it is depicted by French medieval fictional texts. This investigation will show the importance of the theme of human reproduction in the heroic imagination of the Middle Ages. Rites, cults and practices contributed to create a sort of mythological narrative of human birth, dominated by figures of fairies who, in those particular contexts, have a strong maternal and nourishing ethos. This intense ritual and narrative activity is of course to be linked to preoccupations surrounding the survival of the children. In the second part of the article, we focus on Brun de la Montaigne, a curious text dated from the beginning of the $14^{\text {th }}$ century. The strong involvement of the father of the hero in the birth of his long-desired child offers a very interesting representation of male imagination and superstition about human reproduction within a feudal context. 